\title{
Ellis-van Creveld Syndrome and Congenital Heart Defects: Presentation of an Additional 32 Cases
}

\author{
Matthew J. O'Connor • R. Thomas Collins II
}

Received: 27 September 2011/ Accepted: 9 December 2011/Published online: 28 January 2012

(C) Springer Science+Business Media, LLC 2012

We read with interest the recent article in Pediatric Cardiology by Hills et al. [1]: Ellis-van Creveld Syndrome and Congenital Heart Defects: Presentation of an Additional 32 Cases. The authors are to be commended for their work, which sheds further light on the breadth and complexity of congenital heart disease (CHD) seen in patients with Ellis-van Creveld syndrome (EvC).

We agree with the authors that the diagnostic precision afforded by the Pediatric Cardiac Care Consortium (PCCC) database is of great value in its application to EvC because such anatomic detail has not been provided in prior clinical reports. For a syndrome with a 60-70\% incidence of CHD, the detailed focus on CHD in this paper is of value to pediatric cardiologists, cardiovascular surgeons, and other providers involved in the care of children with EvC. With respect to outcomes after CHD surgery for patients with $\mathrm{EvC}$, however, some of the results described by Hills et al. [1] were unclear to us.

We recently reported outcomes of CHD surgery for children with EvC during the recent era in a smaller, mostly Amish, population [2]. Similar to the findings by Hills et al. [1], we noted a relatively high incidence of systemic venous abnormalities (33\%) in our study. However, and in seeming contrast to the data reported by Hills et al. [1], we found that CHD surgery for patients with EvC was associated with a postoperative mortality rate of $44 \%$, with the deaths occurring at a median of 102 days (range, 25-149 days) postoperatively. This incidence is substantially higher than that reported in the study by Hills et al. [1], in which no postoperative deaths were reported. In our

M. J. O'Connor $(\varangle) \cdot$ R. Thomas Collins II

University of Arkansas for Medical Sciences, Arkansas

Children's Hospital, Little Rock, AR 72202, USA

e-mail: mjoconnor@uams.edu study, the majority of deaths were related to respiratory complications, presumably on account of the thoracic dystrophy that is a hallmark of EvC.

It is unclear to us how Hills et al. [1] evaluated followup and postoperative mortality in their series of patients. A major limitation of the PCCC database is incomplete or missing follow-up data [3]. In the study by Hills et al. [1], therefore, what was the duration of the follow-up period? A shorter follow-up period (i.e., 30 days), used frequently to determine postoperative mortality, may not be sufficient to capture the true effect of CHD surgery on the respiratory system in EvC. Although we certainly support the concept of repair or palliation of $\mathrm{CHD}$ in patients with EvC, we stress that surgery for CHD in patients with $\mathrm{EvC}$ is a highrisk undertaking. In our study, substantial mortality was not the only notable finding. In addition, all those surviving surgery experienced postoperative respiratory morbidity (prolonged need for supplemental oxygen, prolonged mechanical ventilation, and tracheostomy). Those involved in the care of children with EvC must consider such data when making treatment decisions and counseling families.

\section{Author Responses}

I appreciate the opportunity to expand on the outcomes for the patients presented in our recent article, Ellis-van Creveld Syndrome and Congenital Heart Defects: Presentation of an Additional 32 Cases. Drs. O'Connor and Collins have reported on an additional cohort of patients who underwent surgery for Ellis-van Creveld syndrome and demonstrated a higher incidence of postoperative mortality, usually secondary to respiratory compromise due to thoracic dystrophy.

The Pediatric Cardiac Care Consortium is indeed limited in terms of the long-term follow-up data available. 
However, the minimum duration of the follow-up period is to the time of discharge from the hospital even if this exceeds the traditional postoperative mortality limit of 30 days. For some patients, additional follow-up data are available, with the reporting of subsequent cardiac catheterizations or surgeries.

Certainly, the patients in our study may represent a somewhat "healthier" subset of patients with Ellis-van Creveld syndrome in that they were considered appropriate candidates for cardiac catheterization or surgery. This is reflected in the fact that none of the patients admitted outside the newborn period in our study were ventilator dependent at the time of catheterization or surgery.

Morbidity also may be indirectly evaluated using hospital length of stay data. A significant proportion of admissions for cardiac catheterization alone $(n=30)$ were for 2 days or less (67\%), with longer stays related to admissions on the day of birth (length of stay range, 1-54 days). As stated in our report, three patients died after cardiac catheterization without additional operative intervention 3 to 45 days subsequent to their initial procedure. Two of these deaths were attributed to nonrespiratory causes (sepsis and new onset of overwhelming aortic insufficiency), whereas one patient died of cardiac arrest and restrictive chest wall disease.

The hospital length of stay for surgery-only admissions ( $n=21$ ) ranged from 4 to 48 days (overall average, 12.9 days). A trend toward shorter hospitalizations has been seen in recent years. In our study, only five admissions were longer than 14 days, and 11 patients were discharged within 7 days after admission. Although discharge summaries are not available in all cases for assessment of the need for home oxygen therapy, it may be surmised that a short hospital stay suggests a lack of need for prolonged mechanical ventilation. An additional four hospitalizations that included multiple catheterizations or surgeries had lengths of stay ranging from 20 to 140 days, with the longest hospitalization related to prematurity, twin gestation, chronic lung disease, and ultimate need for tracheostomy.

The age of the patient at the time of admission also proved to be an important factor in mortality. All three nonsurvivors in our study were younger than 2 months at the time of death. The average age at the time of surgery for all the patients in our study was 2.5 years (range, 5 days to 12 years).

It is certainly agreed that careful consideration of each patient's clinical status must be included in the provision of appropriate presurgical counseling for parents of children with Ellis-van Creveld syndrome. I appreciate the additional cases presented by Drs. O'Connor and Collins and their reminder of the significant morbidities associated with thoracic dystrophy present in this population.

Christine B. Hills

Department of Pediatric Cardiology, University of Minnesota, MMC 94 Delaware St. SE, Minneapolis, MN 55455, USA

\section{References}

1. Hills CB, Kochilas L, Schimmenti LA, Moller JH (2011) Ellis-van Creveld syndrome and congenital heart defects: presentation of an additional 32 cases. Pediatr Cardiol 32:977-982

2. O'Connor MJ, Rider NL, Collins RT, Hanna BD, Morton DH, Strauss KA (2011) Contemporary management of congenital malformations of the heart in infants with Ellis-van Creveld syndrome: a report of nine cases. Cardiol Young 21:145-152

3. Pham PP, Moller JH, Hills C, Larson V, Pyles L (2009) Cardiac catheterization and operative outcomes from a multicenter consortium for children with Williams syndrome. Pediatr Cardiol 30: $9-14$ 